\title{
Assessment of Large Granular Lymphocyte during Dasatinib Therapy and its Relation to Deep Molecular Response in Chronic Myeloid Leukemia
}

\author{
Haiqing Wang', Shupei Ma1
}

\author{
${ }^{1}$ Department of Haematology, Qingdao Municipal Hospital
}

\begin{abstract}
Objective: To compare the differences of deep molecular response (DMR) rate and time of obtaining DMR between dasatinib treated patients with increased and normal levels of large granular lymphocytes (LGLs) in newly diagnosed or imatinib resistant/intolerant chronic myeloid leukemia (CML) patients with positive BCR-ABL fusion gene. Methods: LGLs in peripheral blood were counted by flow cytometry and BCR-ABL fusion gene transcriptional level was detected by real-time quantitative polymerase chain reaction in 25 CML patients before and $1,3,6,9,12,15,18$ months after dasatinib treatment. The enrolled patients were classified into LGLs+ group and LGLs- group according to whether the LGL counts were increased or not. Results: Among the 25 patients investigated, LGLs in 15 cases $(5 / 15,60 \%)$ were increased. Five cases in LGL+ group obtained DMR $(33.3 \%)$ which was significantly higher than that of LGL- group $(33.3 \%$ vs $10 \%, \mathrm{P}<0.05)$. The median time of obtaining DMR in LGLs+ group and LGLs- group were 12 and 15 months respectively. Conclusions: Increased LGLs can be used as an indicator of prognosis in CML patients with positive BCR-ABL fusion gene who treated with dasatinib.
\end{abstract}

Keywords: Chronic Myeloid Leukemia, Large Granular Lymphocyte, Dasatinib, Deep Molecular Response

\section{Introduction}

Chronic myeloid leukemia (CML), represented $15 \%$ of leukemia, was a malignant hematopoietic disease characterized by proliferation of immature granulocytes $^{[1]}$. The occurrence of CML was closely related to the formation of BCR-ABL fusion gene which was produced by translocation of chromosome 9 and 22. BCR-ABL fusion gene was positive in more than $95 \%$ CML patients ${ }^{[2]}$. Moreover, the quantities of BCR-ABL fusion gene expression represented the load of leukemia cells in CML patients. Thus, detection the quantities of BCR-ABL fusion gene could be used to evaluate the effect of treatment in CML patients. Tyrosine kinase inhibitors (TKIs) may prevented proliferation and promote apoptosis of leukemia cells by blocking signal transduction pathways ${ }^{[3]}$. Recently, a variety of studies have shown that dasatinib, one of the second generation TKI agents, may improve the number of lymphocytes in peripheral blood in CML patients $^{[4,5]}$. The increased lymphocytes were mainly recognized as LGLs by morphology and immunophenotype analysis showed that the increased LGLs were mainly NK cells and cytotoxic T cells (CTLs) ${ }^{[4-9]}$. Previous studies have shown that CML patients with increased LGLs might have higher possibility of acquiring deep molecular response $(\mathrm{DMR})^{[10,11]}$. Thus, increased LGLs were regarded as a predictor of favorable treatment response in CML patients ${ }^{[5,12]}$. We analyzed the clinical data of $25 \mathrm{CML}$ patients treated with dasatinib to explore the difference of DMR rate and time of obtaining DMR between dasatinib treated patients with increased and normal levels of LGLs in newly diagnosed or imatinib resistant/intolerant $\mathrm{CML}$ patients with positive BCR-ABL fusion gene.

\section{Patients and Methods}

Patients and treatment

A total of 25 patients diagnosed with CML in Qingdao Municipal Hospital from January 2014 to January 2017 were enrolled in this study and all the enrolled patients were followed up for 18 months. Seven cases were female and 18 cases were male. The median age of these patients was 51 (ranged from19 to 77) years old. Five of the 25 patients enrolled were newly diagnosed and the other 20 patients were intolerant or resistant to imatinib. Twenty patients were in chronic phase (CP) and the other 5 were in accelerated phase (AP). There was no death or disease progression in all the 25 patients investigated during the follow up period.

All patients enrolled were treated by orally taken the second generation TKI agents-dasatinib. Patients in CP took 100mg once daily and those in AP took 70mg twice daily. 
Assessment of treatment response

Peripheral blood samples of all the enrolled patients were taken before and 1, 3, 6, 9, 12, 15, 18 months after the starting of dasatinib treatment. Lymphocyte subsets, mainly LGLs (NK cells and Cytotoxic T cells) were counted by flow cytometry. The enrolled patients were classified into LGLs+ group and LGLs- group according to whether the LGL counts were increased or not. The quantity of BCR/ABL transcript was detected by real-time quantitative polymerase chain reaction. DMR was defined as less than $0.01 \%$ of the International Scale (BCR-ABL $\left.{ }^{\text {is }}\right)^{[13]}$.
Statistical analysis

Pearson's chi-square $\left(\chi^{2}\right)$ test and Fisher's exact test were used to analyze the data in this study. All the analyses were conducted using SPSS 19.0 software. P $<0.05$ was regarded as statistically significant.

\section{Results}

\section{DMR in newly diagnosed CML patients}

Three out of the 5 newly diagnosed CML patients achieved DMR, including 2 male (1 in CP, 1 in AP) and 1 female in CP. The time of obtaining DMR after dasatinib treatment was 3 and 12 months for the two patients in $\mathrm{CP}$ and 15 months for the patient in AP (Table 1).

Table1 DMR in newly diagnosed CML patients

\begin{tabular}{rcccc}
\hline Patients & Age(year) & Gender & Phase & DMR (month) \\
\hline 1 & 51 & male & AP & $+(15)$ \\
2 & 59 & female & CP & $+(3)$ \\
3 & 46 & male & CP & $+(12)$ \\
4 & 65 & female & CP & - \\
5 & 45 & male & CP & - \\
\hline
\end{tabular}

\section{DMR in CML patients resistant or intolerant to} imatinib

Twenty CML patients who were resistant or intolerance to imatinib were treated with dasatinib. Three cases $(2$ cases of male and 1 case of female) achieved DMR and all of them were in CP. The time of obtaining DMR was 6, 12 and 15 months after the treatment of dasatinib respectively. None of the 4 patients in AP achieved DMR (Table 2).

Table 2 DMR in CML patients resistant or intolerant to Imatinib

\begin{tabular}{ccccc}
\hline Patients & Age $($ year $)$ & Gender & Phase & DMR (month) \\
\hline 1 & 28 & female & $\mathrm{CP}$ & - \\
2 & 47 & male & $\mathrm{CP}$ & $+(12)$ \\
3 & 43 & male & $\mathrm{CP}$ & - \\
4 & 66 & female & $\mathrm{CP}$ & $+(15)$ \\
5 & 51 & male & $\mathrm{CP}$ & - \\
6 & 70 & male & $\mathrm{CP}$ & - \\
7 & 34 & male & $\mathrm{CP}$ & $+(6)$ \\
8 & 54 & male & $\mathrm{CP}$ & - \\
9 & 57 & male & $\mathrm{CP}$ & - \\
10 & 47 & male & $\mathrm{AP}$ & - \\
11 & 66 & male & $\mathrm{CP}$ & - \\
12 & 52 & male & $\mathrm{AP}$ & - \\
13 & 77 & male & $\mathrm{CP}$ & - \\
14 & 27 & male & $\mathrm{CP}$ & - \\
15 & 72 & female & $\mathrm{AP}$ & - \\
16 & 44 & female & $\mathrm{AP}$ & - \\
17 & 59 & male & $\mathrm{CP}$ & - \\
18 & 46 & male & $\mathrm{CP}$ & - \\
19 & 41 & male & $\mathrm{CP}$ & - \\
20 & 19 & female & $\mathrm{CP}$ & - \\
\hline
\end{tabular}




\section{Correlation between LGLs and DMR}

Fifteen patients who had increased LGLs after dasatinib treatment were classified as LGL+ group. Five of them $(33.3 \%)$ achieved DMR. The other 10 patients who had normal LGLs after dasatinib treatment were classified as LGL- group. Only 1 of the 10 patients $(10 \%)$ achieved DMR. The incidence of DMR between LGL+ group and LGL-group was significantly different (fisher's exact test, $\mathrm{P}=0.034$ ) (Table 3).

Table 3 DMR in LGL+ and LGL- groups

\begin{tabular}{llll}
\hline Groups & DMR (+) & DMR (-) & Total (n) \\
\hline LGL+ & $5(33.33 \%)$ & $10(66.66 \%)$ & 15 \\
\hline LGL- & $1(10 \%)$ & $9(90 \%)$ & 10 \\
\hline Total $(\mathbf{n})$ & 6 & 19 & 25 \\
\hline
\end{tabular}

The relationship between LGL and BCR-ABL fusion gene transcriptional level in LGL+ group Fifteen of $25(60 \%)$ patients had increased LGLs after dasatinib treatment. Six and seven of them had increased counts of NK cells and CTLs respectively. Two cases had increased counts of both NK cells and
CTLs.

The patients in LGL+ group had increased counts of NK cells and CTLs after dasatinib treatment (Table 4), and both of them were negatively correlated with the level of $\mathrm{BCR}-\mathrm{ABL}^{\text {is }}(\mathrm{NK}$ : $\mathrm{r}=-0.112, \mathrm{P}<0.05$; $\mathrm{CTL}$ : $\mathrm{r}=-0.343, \mathrm{P}<0.05)$.

Table 4 The counts of NK cells, CTLs and level of BCR-ABL ${ }^{\text {is }}$ in LGL+ group before and after dasatinib treatment

\begin{tabular}{lllll}
\hline Group & Treatment & $\begin{array}{l}\text { NK cells }\left(\mathbf{1 0}^{\mathbf{9}} / \mathbf{L}\right) \\
(\mathbf{C D 1 6 + C D 5 6 + )}\end{array}$ & $\begin{array}{l}\text { CTLs }\left(\mathbf{1 0}^{\mathbf{9}} / \mathbf{L}\right) \\
(\mathbf{C D 8}+\mathbf{C D 2 8 +})\end{array}$ & BCR-ABL $^{\text {is }}(\%)$ \\
\hline LGL+ & Before & $0.57 \pm 0.06$ & $0.06 \pm 0.01$ & $142.2 \pm 41.9$ \\
& After & $1.81 \pm 1.03$ & $0.21 \pm 0.13$ & $2.41 \% \pm 2.2$ \\
\hline
\end{tabular}

\section{Median time of achieving DMR after dasatinib treatment}

The median time of obtaining DMR in LGLs+ group and LGL- group were 12 and 15 months respectively.

\section{Discussion}

Studies have shown that the first generation TKIs imatinib could target BCR-ABL fusion gene and significantly improved progression free survival (PFS) and overall survival (OS) in CML patients ${ }^{[14-15]}$. Compared to imatinib, the second generation TKI agent dasatinib had an enhanced effect on BCR-ABL fusion gene ${ }^{[16]}$. It has been reported that the affinity between dasatinib and tyrosine kinase is 100 times higher than that of imatinib ${ }^{[17]}$.

The defects of T lymphocytes and NK cells play an important role in the occurrence and development of hematological malignant diseases ${ }^{[18,19]}$. T lymphocytes take part in the regulation of tumor immunity ${ }^{[20]}$, while NK cells can nonspecifically kill malignant leukemia cells. Immune deficiency of T cells is common in CML patients, and the immune function of CML patients is closely related to the disease progression of CML. Therefore, observing the changes of $\mathrm{T}$ lymphocyte subsets and NK cells in peripheral blood is valuable to monitor the progression of CML patients. The transcriptional level of BCR-ABL fusion gene reflects the severity of CML patients ${ }^{[21]}$, which is of great importance to the treatment of CML. Therefore, detection BCR-ABL fusion gene transcriptional level is particularly important to evaluate the therapeutic efficacy and prognosis of CML patients.
Powers et al. ${ }^{[7]}$ discovered that $31 \%(5 / 16)$ CML patients showed LGL lymphocytosis (increased LGLs) after dasatinib treatment. Nagata et al.'s study [6] showed that the LGL lymphocytosis in peripheral blood appeared in 9 out of 20 patients with CML who were continuously treated with dasatinib during the follow-up period. They also demonstrated that LGL lymphocytosis might correlate with obtaining molecular response (MR). Tanaka et al. ${ }^{[8]}$ showed that 15 of 25 CML patients who was treated with dasatinib had LGL lymphocytosis in peripheral blood. Of which, the counts of NK cells and CTLs increased in 15 and 11 cases respectively. And LGL lymphocytosis might relate to achieving complete molecular response (CMR). In this study, we showed that increased LGLs stimulated by dasatinib were associated with high rate of DMR and short time of achieving DMR in CML patients. These results were in accordance with the abovementioned studies and suggested that increased LGLs can be used as an indicator of prognosis in CML patients who treated with dasatinib. It has been reported that dasatinib stimulated immune reaction in the formation of LGL lymphocytosis in peripheral blood of CML patients ${ }^{[22]}$. The precise mechanism of LGL proliferation during the dasatinib treatment is still unclear. It is presumed that dasatinib could activate or modulate the proliferation and function of NK cells.

In this study, we showed that increased LGLs can be used as an indicator of prognosis in CML patients with positive BCR-ABL fusion gene who treated with dasatinib. Due to the limited number of patients 
enrolled in this study, we expected further studies with larger sample size to verity our results.

\section{References}

1. National Comprehensive Cancer Network. NCCN Clinical Practice Guidelines in Oncology: Chronic Myelogenous Leukemia, V.2.2008. NCCN Web Site. 8-28-2007. Accessed October 25, 2007.

2. Zhang A, Hu Q, Zhang L, et al. Clinical analysis of children diagnosed with BCR/ABL positive acute lymphoblastic leukemia. Leukemia. Lymphoma 2014, 23 (3):160-162.

3. Zhou B, An C, Pu X. Research and development status of next generation tyrosine kinase inhibitor agents. Chinese Journal of New Drugs 2011, 20(7):1661-1666

4. Mustjoki S, Ekblom M, Arstila, TP, et al. Clonal expansion of T/NK-cells during tyrosine kinase inhibitor dasatinib therapy. Leukemia 2009, 23:1398-1405.

5. Kim DH, Kamel-Reid S, Chang H, et al. Natural killer or Natural killer/T cell lineage large granular lymphocytes associated with dasatinib therapy for Philadelphia chromosome positive leukemia. Haematologica 2009, 94: 135-139.

6. Nagata Y, Ohashi K, Fukuda S, et al. Clinical features of dasatinib-induced large granular lymphocytosis and pleural effusion. Int J Hematol 2010, 91:799-807.

7. Powers JJ, Dubovsky JA, Epling-Burnette PK, et al. A molecular and functional analysis of large granular lymphocyte expansions in patients with chronic myelogenous leukemia treated with tyrosine kinase inhibitors. Leuk Lymphoma 2015, 2:668-679.

8. Tanaka H and Nakashima S. Rapid and sustained increase of large granular lymphocytes and rare cytomegalovirus reactivation during dasatinib treatment in chronic myelogenous leukemia patients. Int J Hematol 2012, 96:308-319.

9. Paydas S. Dasatinib, large granular lymphocytosis, and pleural effusion: Useful or adverse effect? Crit Rev Oncol Hematol 2014, 89:242-247.

10. Kumagai T, Matsuki E, Inokuchi K, et al. Relative increase in lymphocytes from as early as 1 month predicts improved response to dasatinib in chronic-phase chronic myelogenous leukemia. Int J Hematol 2014, 99:41-52.

11. Inokuchi K, Kumagai T, Matsuki E, et al. Efficacy of molecular response at 1 or 3 months after the initiation of dasatinib treatment can predict an improved response to dasatinib in imatinib-resistant or imatinib-intolerant Japanese patients with chronic myelogenous leukemia during the chronic phase. J Clin Exp Hematol 2014, 54:197-204.

12. Lee SJ, Jung CW, Kim DY, et al. Retrospective multicenter study on the development of peripheral lymphocytosis following second-line dasatinib therapy for chronic myeloid leuk-mia. Am J Hematol 2011, 86:346-350.

13. Iriyama $\mathrm{N}$, Fujisawa $\mathrm{S}$, Yoshida $\mathrm{C}$, et al. Early cytotoxic lymphocyte expansion contributes to a deep molecular response to dasatinib in patients with newly diagnosed chronic myeloid leukemia in the chronic phase: results of the D-first study. Am J Hematol 2015, 90:819-824.

14. Falchi L, Kantarjian HM, Wang X, et al. Significance of deeper molecular responses in patients with chronic myeloid leukemia in early chronic phase treated with tyrosine kinase inhibitors. Am J Hematol 2013, 88 (12):1024-1029.

15. Savona MR and Saglio G. Identifying the time to change BCR-ABL inhibitor therapy in patients with chronic myeloid leukemia. Acta Haematol 2013, 130 (4):268-278.

16. Shan NP, Tran C, Lee FY et al. Overriding imatinib resistance with a novel ABL kinase inhibitor. Science 2004, 305 (5682): 399-401.

17. Guo F, Chen Y, Yang M et al. Therapeutic effect of dasatinib on chronic myelogenous leukemia patients in acceleration stage. Chinese Journal of Disease Control 2015, 19(6):601-604.

18. Qing H, Shi H, Liu Z et al. Peripheral blood T lymphocyte subgroup detection and clinical significance in 253 patients diagnosed with malignant tumor. Laboratory Diagnostics of China 2009, 13(11):1589-1590.
19. Wu $\mathrm{X}$, Zhang $\mathrm{Q}$, Hou $\mathrm{S}$ et al. Detection and clinical significance of peripheral blood $\mathrm{T}$ lymphocyte subgroup and natural killer cell activity on refractory lymphoma patients. Leukemia. Lymphoma 2011, 20(3):162-164.

20. Zhu J, Yamane H, Paul WE. Differentiation of effector CD4 T cell populations. Annu Rev Immunol 2010, 28:445-489.

21. Yao Z, Zhao G, Wang, Y, et al. Analysis of the relationship between clinical status and quantitative of BCR/ABL in CML patients. Chinese Journal of Experimental Hematology 2009, 17(4):861-865.

22. Qiu Z, Xu W, Li J. Large granular lymphocytosis during dasatinib therapy. Cancer Biology \&Therapy 2014, 15: 247-255. 